\title{
Simple Modelling to Calculate the Parasitic Components for the Materials in AC Circuit
}

\author{
Samy Khalil Kamel Shaat \\ Department of Physics, Islamic University, Gaza, P.O. Box 108, Palestine. \\ Correspondence should be addressed to Samy K. K. Shaat; samyshaat@yahoo.com
}

\begin{abstract}
The main goal of this work is to find a simple mathematical model to calculate parasitic components for the materials in AC circuit. There are different methods such as Delay-Time, Lissajous, Product and Curve-fitting that can be performed to study the parasitic properties. Among them, the Delay-Time method can be achieved to find the phase shift $(\varphi)$ between any two different sine waves using an oscilloscope. The $\varphi$ was exercised to resolve the parallel model for the examined sample in AC circuit. The phasor diagram of the parallel sample model in AC circuit was plotted. All the fundamental and derived quantities of the AC circuit can be calculated, such as the immittances, conductivity and dielectric parameters. The presented method is considered as a simple method comparing to others. Therefore, it can be replaced the others as well as it can be used from the scientists and engineers.
\end{abstract}

Keywords - AC Circuit, Phase sift, parallel Diagram.

\section{INTRODUCTION}

The significance of study the electrochemistry has moved from a time/focused dependency to frequency-related phenomena, a trend toward alternating current (AC) small-signal studies [1]. The importance of such studies is to calculate various quantities such as the impedance, immittances, admittance, modulus, dielectric permittivity, and conductivity. The effect of polycrystallinity on these quantities for solid dielectric materials were reported in refs. [2-4]. Nevertheless, the problem is the measuring of the impedance for these materials. However, the study of the AC properties for the materials can be launch using different methods. Based on somewhat oversimplified physics model; the sample can be dealt as a two parallel plat capacitor separated by examined material) as shown in Figure 1, which can be considered as a capacitor with double electrical layers (DELs). DELs and their inherent capacitive reactance $\left(X_{C}\right)$ are well-known by the distribution of their relaxation times [1].

The variation ultimately of the electrical response of the sample, which can be considered as a heterogeneous cell, depends on the species of charge present, the microstructure, electrolyte, the texture and nature of the plates [1,4]. The most significant properties of the sample are the capacitance and parasitic (stray). The property effects on the AC circuit behaviour such as: aan increase in propagation delay or equivalently, b- a drop in performance, c- an introduction of extra noise sources, which affects the reliability of the circuit, and d- an impact on the energy dissipation and the power distribution [5]. Both the speed/frequency and dynamic power dissipation of a circuit are affected by propagation delay, so timing analysis has been investigated for several decades [6-12].

As seen in Figure 1, the sample with its parasitic components can be represented as LCR parallel. Therefore, Figure 2 shows that, the sample is introduced in a half bridge configuration with the sine wave function generator. The self-inductance (L) and Ohmic resistance (R) depend, strongly, on the physical structure. Such as, the Ohmic resistance of the electrolyte is the only influencing factor in the impedance of the cell, as it is not affected by frequency [13]. Additionally, the dielectric plays a major role, which is represented as an Ohmic resistance. Moreover, the electrolyte in electrolytic capacitors has an Ohmic resistance. This work presents the anatomy of sample in a parallel AC circuit to calculate the $X_{C}^{p}$ and the equivalent resistance $\left(R_{E}^{p}\right)$, and to estimate other quantities, as well. Herein, the other parasitic L did not present which may be needed another measurement method. However, the effects of the $\mathrm{L}$ are noticeable as spikes in the oscilloscope image.

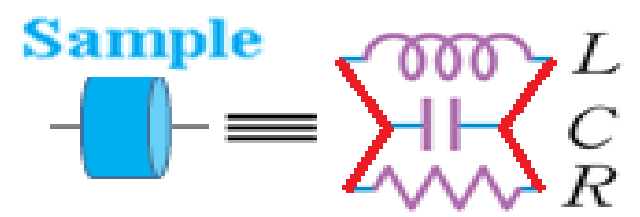

Figure 1: Parasitic model of the sample in AC circuit. 


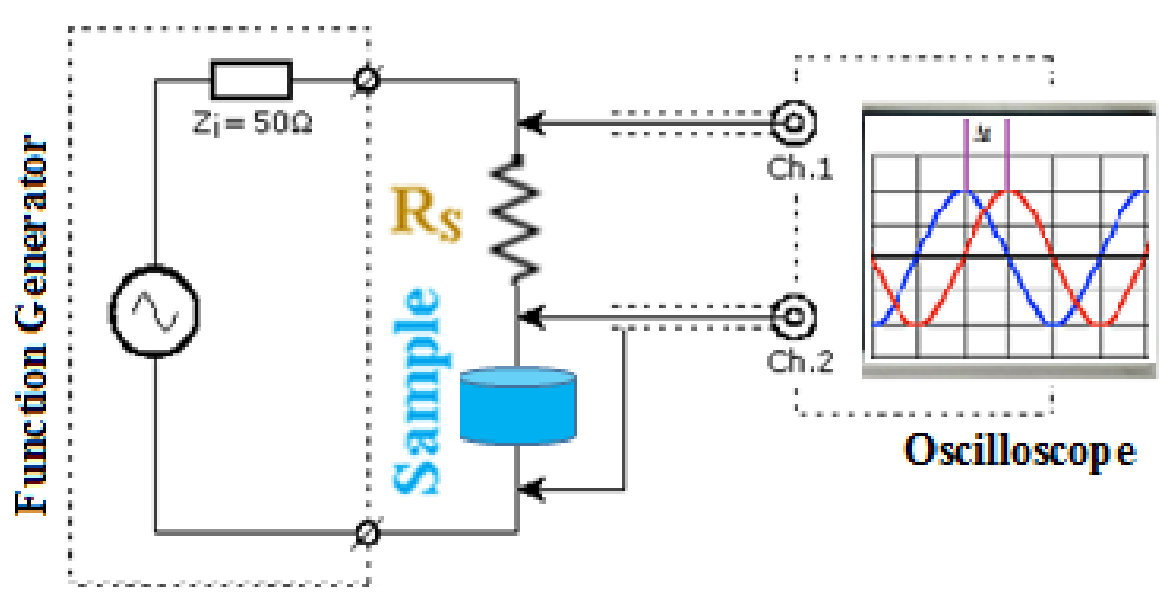

Figure 2: Examined sample in AC circuit.

\section{MATERIALS AND METHODS}

\section{Measurements of the Phase difference for sinusoidal waves.}

It is well known that, the phase difference or shift $(\varphi)$ can be produced between any two different waveforms for the AC circuit. Subsequently, different methods and techniques can be used to measure the $\varphi$ between more than one waveforms using an oscilloscope. These methods are Delay-Time, Lissajous, Product and Curve-fitting as shown in Table 1. In addition, the advantages and limitations of each are in Table (1) [14,15]. It is noticed from this table that, among these methods the DelayTime is considered the best one to calculate the $\varphi$.

As shown in Figure 2, $\varphi$ can be calculated by measuring the Delay-time $(\Delta t)$ between two different sine waves using the following formula:

$$
\varphi=2 \pi f \Delta t
$$

where $f$ is the linear frequency for the sine wave.

As revealed in the Figure 3, the circuit consists of a sample and a resistor $\left(R_{S}\right)$. This circuit, can be performed to calculate $X_{C}^{p}$, $R_{E}^{p}$ and other derived parameters. Here the subscript $p$ stands for parallel. To resolve the assumed circuit in Figure 3, phasors diagram should be plotted, which are exhibited in Figures $4 \mathrm{a}$ and $4 \mathrm{~b}$. The currents and voltages phasors diagram are shown in Figures $4 \mathrm{a}$ and $4 \mathrm{~b}$, respectively. After measuring the peak voltage of the applied voltage $\left(V_{T}^{p}\right)$, the peak voltage of the $R_{S}\left(V_{R}^{S}\right)$ and $\Delta t$ by using oscilloscope different quantities can be found such as the total currents $\left(I_{T}^{p}\right)$ and, consequently, the voltage a cross the sample $\left(V_{S}^{p}\right)$ and the phase angles $(\delta, \varphi$ and $\alpha)$ as shown in Figure 4a.

Table 1: Comparison between different methods for measuring phase difference.

\begin{tabular}{|c|c|c|c|}
\hline Methods & Waveform & Advantages & Limitations \\
\hline Delay-Time & All waveforms. & - Works with nonsinusoidal wave-forms. & $\begin{array}{l}\text { - Errors introduced when DC offsets are } \\
\text { present. }\end{array}$ \\
\hline Product & Sine wave only. & $\begin{array}{l}\text { - No manual reading of values from display (can } \\
\text { be automated). }\end{array}$ & $\begin{array}{l}\text { Errors introduced when DC offsets are } \\
\text { present. } \\
\text { - } \text { Reduced precision near } \varphi=0^{\circ} \text { and } 180^{\circ} \text {. }\end{array}$ \\
\hline Lissajous & Sine wave only. & $\begin{array}{l}\text { - } \quad \text { No need to measure time scales. } \\
\text { - Cool. }\end{array}$ & 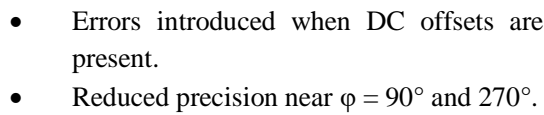 \\
\hline Curve-fitting & All waveforms. & $\begin{array}{l}\text { - No manual reading of values from display (can } \\
\text { be automated). } \\
\text { - Works with nonsinusoidal wave-forms. } \\
\text { - Uses entire waveform information to increase } \\
\text { accuracy. } \\
\text { Cool. }\end{array}$ & - Possible incorrect solution. \\
\hline
\end{tabular}




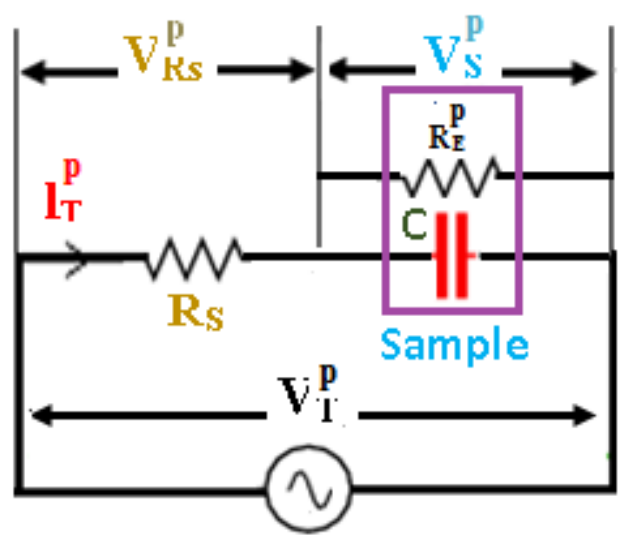

Figure 3: The sample connected in an AC circuit of a half bridge configuration.

(a)

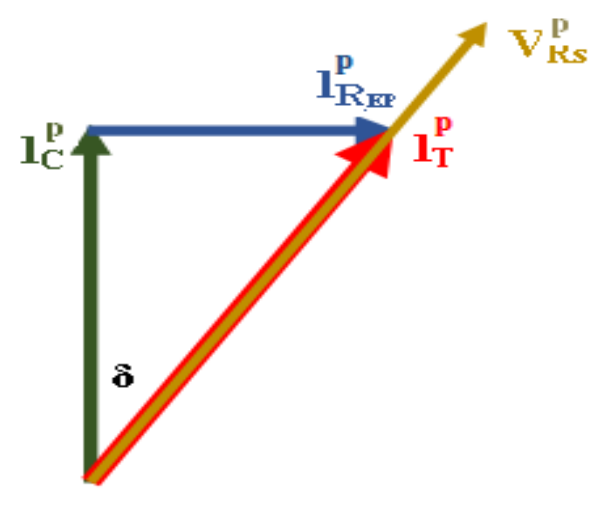

(b)

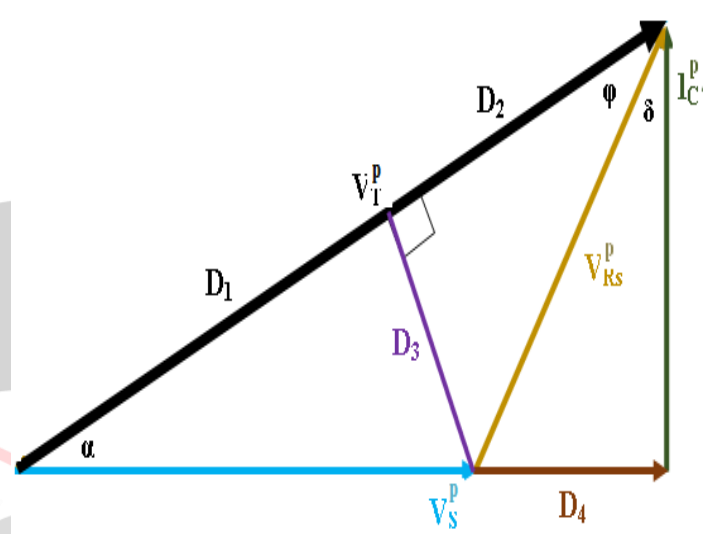

Figure 4: Phasor diagram for AC circuit in Figure 3 (a) currents and (b) voltages.

Suppose that $v_{R S}^{p}$ is the instantaneous voltage cross $R_{S}$ and $v_{S}^{p}$ is the instantaneous voltage cross the sample both oscillate harmonically in time as,

$$
\begin{gathered}
v_{R S}^{p}=V_{R S}^{P} \sin (\omega t+\varphi)=V_{R S}^{P}(\sin \omega t \cos \varphi+\cos \omega t \sin \varphi) \\
v_{S}^{p}=V_{S}^{p} \sin (\omega t-\alpha)=V_{S}^{p}(\sin \omega t \cos \alpha-\cos \omega t \sin \alpha)
\end{gathered}
$$

where $V_{R S}^{P}$ and $V_{S}^{p}$ are the peak voltage of the $v_{R S}^{p}$ and $v_{S}^{p}$, respectively.

By adding the right hand sides of Eqs. 2 and 3, then rearrange the terms one can find

$$
v_{R S}^{p}+v_{S}^{p}=\sin \omega t\left(V_{R S}^{P} \cos \varphi+V_{S}^{p} \cos \alpha\right)+\cos \omega t\left(V_{R S}^{P} \sin \varphi-V_{S}^{p} \sin \alpha\right)
$$

But the applied voltage $\left(v_{T}^{p}\right)$ equals to

$$
v_{T}^{p}=v_{R S}^{p}+v_{S}^{p}
$$

In general, $v_{T}^{p}$ can be written as follows

$$
v_{T}^{p}=V_{T}^{p} \sin (\omega t+\beta)
$$

with $V_{T}^{p}$ is the peak voltage of the $v_{T}^{p}$ and $\beta$ is a phase angle, which can be rewritten by

Compare Eq. 7 with Eq. 5, one can find that

$$
v_{T}^{p}=V_{T}^{p} \sin \omega t \cos \beta+V_{T}^{p} \cos \omega t \sin \beta
$$

$$
\begin{aligned}
& V_{T}^{p} \cos \beta=V_{R S}^{P} \cos \varphi+V_{S}^{p} \cos \alpha \\
& V_{T}^{S} \sin \beta=V_{R S}^{P} \sin \varphi-V_{S}^{p} \sin \alpha
\end{aligned}
$$

Square both sides of Eqs. 8 and 9, implies to

$$
\begin{gathered}
\left(V_{T}^{p} \cos \beta\right)^{2}=\left(V_{R S}^{P} \cos \varphi\right)^{2}+2 V_{R S}^{P} V_{S}^{p} \cos \varphi \cos \alpha+\left(V_{S}^{p} \cos \alpha\right)^{2} \\
\left(V_{T}^{p} \sin \beta\right)^{2}=\left(V_{R S}^{P} \sin \varphi\right)^{2}-2 V_{R S}^{P} V_{S}^{p} \sin \varphi \sin \alpha+\left(V_{S}^{p} \sin \alpha\right)^{2}
\end{gathered}
$$

Adding the left hand side and the right hand side of Eqs. 10 and 11, then one can get $V_{T}^{p}$

$$
V_{T}^{p}=\sqrt{\left(V_{R S}^{P}\right)^{2}+2 V_{R S}^{P} V_{S}^{p} \cos (\alpha+\varphi)+\left(V_{S}^{p}\right)^{2}}
$$

Again, from Figure 4, the summation of phases angles is given by

$$
\varphi+\alpha+\delta=90^{\circ}
$$


This implies to

Therefore, Eq. 12 becomes

$$
\cos (\varphi+\alpha)=\cos \left(90^{\circ}-\delta\right)=\sin \delta
$$

$$
V_{T}^{p}=\sqrt{\left(V_{R S}^{P}\right)^{2}+2 V_{R S}^{P} V_{S}^{p} \sin \delta+\left(V_{S}^{p}\right)^{2}}
$$

In straightforward, to find the loss tangent $(\tan \beta)$ divide Eqs. 9 and 8 ,

$$
\tan \beta=\frac{V_{R S}^{P} \sin \varphi-V_{S}^{p} \sin \alpha}{V_{R S}^{P} \cos \varphi+V_{S}^{p} \cos \alpha}
$$

Thus, the phase angle $(\beta)$, is given by

$$
\beta=\tan ^{-1}\left(\frac{V_{R S}^{P} \sin \varphi-V_{S}^{p} \sin \alpha}{V_{R S}^{P} \cos \varphi+V_{S}^{p} \cos \alpha}\right)
$$

In the case of $\beta=0$, implies to $\cos \beta=1$, thus Eq. 7 becomes

$$
V_{T}^{p}=V_{R S}^{P} \cos \varphi+V_{S}^{p} \cos \alpha
$$

and so $\sin \beta=0$, thus Eq. 10 becomes

$$
V_{R S}^{P} \sin \varphi=V_{S}^{p} \sin \alpha
$$

By using Eq. 19, substitute $V_{R S}^{P}$ into Eq. 18, which implies to

$$
\begin{aligned}
& V_{T}^{p}=\frac{V_{S}^{p}}{\sin \varphi}(\sin \alpha \cos \varphi+\sin \varphi \cos \alpha)=\frac{V_{S}^{p} \sin (\alpha+\varphi)}{\sin \varphi} \\
& V_{T}^{p}=V_{S}^{p}(\sin \alpha \cot \varphi+\cos \alpha)
\end{aligned}
$$

Again, from Eq. 13,

$$
\sin (\varphi+\alpha)=\sin \left(90^{\circ}-\delta\right)=\cos \delta
$$

As a result, Eq. 20 becomes

$$
V_{T}^{p}=\frac{V_{S}^{p} \cos \delta}{\sin \varphi}
$$

Based on the AC circuit (Figure 3), which is a circuit that is driven by a voltage source (emf) that oscillates harmonically in time as

$$
v_{T}^{p}=V_{T}^{p} \sin \omega t
$$

In the physical regime, where non-linear effects can be neglected, the response is linear. Thus, aside from transients, the current, also, oscillates harmonically, and takes the form

$$
i_{T}^{p}=I_{T}^{p} \sin (\omega t+\varphi)
$$

The amplitude $I_{T}^{p}$ and phase $\varphi$ can be determined from the driving voltage and the nature of the circuit. The amplitude of the current is linearly related to the amplitude of the voltage, where the phase angle is determined by a trigonometric equation. These relations can be very conveniently combined into a single linear relation by expressing the voltage and current as the real parts of complex quantities

$$
\begin{gathered}
v_{T}^{c p}=V_{T}^{c p} e^{j \omega t} \\
i_{T}^{c p}=I_{T}^{p c} e^{j \omega t}
\end{gathered}
$$

with $V_{T}^{p c}=V_{T}^{p}$ and $I_{T}^{p c}=I_{T}^{p} e^{-j \varphi}$ is a complex number.

Once more, based on the phasor diagram in Figure $4 \mathrm{a}$, the $\varphi$ between the two voltages waves $V_{T}^{p}$ and $V_{R s}^{p}$ can be calculated by using Eq. (1). Herein, $I_{T}^{p}$ can be determined by measuring $V_{R S}^{p}$ across the known $R_{S}$ and, also, by applying Ohm's law as follows

$$
I_{T}^{p}=\frac{V_{R S}^{P}}{R_{S}}=\frac{V_{S}^{p}}{Z_{S}^{c p}}=\frac{V_{T}^{p}}{Z_{T}^{c p}}
$$

where $Z_{S}^{c p}$ is the impedance of the sample and $Z_{T}^{c p}$ is the total impedance. Referring to Figure $4 \mathrm{a}$, the vector sum of the $V_{S}^{p}$ is donated by

$$
V_{S}^{p}=D_{1}^{2}+D_{3}^{2}=\left(V_{T}^{p}-D_{2}\right)^{2}+D_{3}^{2}
$$

Geometrically, $D_{1}=V_{S}^{p} \cos \alpha, D_{2}=V_{R S}^{P} \cos \varphi$ and $D_{3}=V_{R S}^{P} \sin \varphi$. Now, substitute these parameters into Eq. 29 to get

$$
V_{S}^{p}=\sqrt{\left(V_{T}^{p}-V_{R S}^{P} \cos \varphi\right)^{2}+\left(V_{R S}^{P} \sin \varphi\right)^{2}}
$$

Now, the phase angle $(\alpha)$ between the two voltage waves $V_{T}^{p}$ and $V_{S}^{p}$ (Figure 4a), can be expressed as follows

$$
\alpha=\sin ^{-1}\left[\frac{\left(V_{R S}^{P} \sin \varphi\right)}{V_{S}^{p}}\right]
$$

From Eq. 13 and Eq. 31, the phase angle $(\delta)$ can be written by 


$$
\delta=\left[90^{\circ}-\left(\varphi+\sin ^{-1}\left[\frac{\left.V_{R S}^{P} \sin \varphi\right)}{V_{S}^{p}}\right]\right)\right]
$$

Because the sample in the circuit is presented as a capacitor and a parallel resistor as in Figure 3, the $V_{S}^{p}$ and the voltage a cross the parallel resistor $\left(V_{R E}^{P}\right)$ have the same value i.e.

Using the Figure 4a, to find,

$$
V_{S}^{p}=V_{R E}^{p}=I_{R E}^{p} R_{E}^{p}
$$

$$
I_{E}^{p}=I_{T}^{P} \sin \delta
$$

Thus, $R_{E}^{S}$ can be found from the following relation

$$
R_{E}^{p}=\frac{V_{E}^{p}}{I_{T}^{P} \sin \delta}=\frac{V_{S}^{p}}{I_{T}^{P} \sin \delta}
$$

Herein, in this supposed model (Figure 3), a capacitor and a resistor are in a parallel connection, therefore,

Referring to the Figure 4a,

$$
V_{S}^{p}=V_{C}^{P}=I_{C}^{P} X_{S}^{p}
$$

$$
I_{C}^{P}=I_{T}^{P} \cos \delta
$$

As a result, the capacitive reactance $\left(X_{S}^{p}\right)$ can be found by

$$
X_{C}^{p}=\frac{V_{S}^{p}}{I_{T}^{p} \cos \delta}=\left[\omega C_{S}^{p}\right]^{-1}
$$

Now, the capacitance of the sample can be calculated by using the following

$$
C_{S}^{p}=\frac{I_{T}^{p} \cos \delta}{\omega V_{S}^{p}}
$$

\section{Immitances functions}

It is worthy to mention that, there are several quantities are called immittances: the impedance $(Z)$, admittance $(Y)$, modulus $(M)$ and dielectric constant or dielectric permittivity $(\varepsilon)$. Where all of them are linked to each other's.

\section{The impedance function}

Impedance $(Z)$ was defined for discrete systems and for arbitrary distributed systems such as the Fourier transform (FFT) of the differential equation defining, the voltage response divided by the FFT of the periodic current excitation [1]. Nevertheless, FFT only decreases differential equations to simple Ohm's law-like form under conditions of linearity, causality, and stationarity of the system; consequently, $Z$ is, properly, defined only for systems fitting these conditions. In the AC circuit, $Z$ has a more general conception than resistance $(R)$ because it considers phase differences. It becomes a fundamental and essential concept in different engineering and science applications.

Based on Eqs. 27 and 28, the impedance ( $Z$ ) can be defined as the ratio of the complex voltage and current amplitudes

$$
Z^{c p}=\frac{V_{T}^{c p}}{I_{T}^{c p}}=\frac{V_{T}^{c p}}{I_{T}^{p}} e^{j \varphi}=Z_{T}^{c p} e^{j \varphi}
$$

where $Z^{c p}$ is usually complex. The complex voltage $V_{T}^{c p}$ and current $I_{T}^{c p}$, thus obey the linear relation,

$$
V_{T}^{c p}=Z^{c p} I_{T}^{c p}
$$

which is a complex generalization of Ohm's law, $V=I R$. The $\mathrm{Z}$ is most directly interpreted when written in polar form, $Z^{c p}=\left|Z^{c p}\right| e^{j \varphi}$. The magnitude $\left|Z^{c p}\right|=V_{T}^{c p} / I_{T}^{c p}$ is called the reactance, and it determines the real amplitude of the current given the real amplitude of the voltage. The phase $\varphi$ of $Z^{c p}$ encodes the phase relation between voltage $v_{T}^{p}=V_{T}^{p} \sin \omega t$ and current $i_{T}^{p}=I_{T}^{p} \sin (\omega t+\varphi)$. Thus, there are many methods to find the impedance of the sample $Z_{S}^{c p}$, Here subscription $\mathrm{s}$ stands for sample, which is expressed in a complex vector, as follows:

$$
Z_{S}^{c p}=Z^{p^{\prime}}+j Z^{p^{\prime \prime}}
$$

where $Z^{p \prime}$ and $Z^{p \prime \prime}$ are the real and imaginary parts of the $Z_{S}^{c p}$, respectively. The three quantities $Z_{S}^{c p}, Z^{p \prime}$ and $Z^{p \prime \prime}$ can be represented in the plane with either rectangular or polar coordinates. In the simplest analysis the $Z_{S}^{c p}$ of the parallel $R C$ circuits model. Herein, the electrical configurations will be encountered in measurement and interpretation of experimental $Z$ spectroscopic behaviour of the double-layer at electrode interfaces.

Experimentally, depend on the Figure $4 \mathrm{~b}$ and Eq. $42, Z^{c p}=Z_{S}^{c p}$ is represented in Figure 5, which can be written by the following:

$$
\frac{1}{Z_{S}^{c p}}=\frac{1}{R_{E}^{p}}+\frac{1}{j X_{S}^{p}}
$$


In order to distinct the real and imaginary components of impedance in this equation multiply the numerator and denominator by the rationalizing complex conjugate of $X_{S}^{p}+j R_{E}^{p}$, viz $X_{S}^{p}-j R_{E}^{p}$, which is giving by

$$
Z_{S}^{c p}=\frac{j R_{E}^{p} X_{S}^{p}}{R_{E}^{p}+j X_{S}^{p}}=\frac{j R_{E}^{p} X_{S}^{p}\left(R_{E}^{p}-j X_{S}^{p}\right)}{\left(R_{E}^{p}+j X_{S}^{p}\right)\left(R_{E}^{p}-j X_{S}^{p}\right)}
$$

So that, $Z_{S}^{c p}$ can be given by

$$
\begin{gathered}
Z_{S}^{c p}=\frac{R_{E}^{p}\left(X_{S}^{p}\right)^{2}-j X_{S}^{p}\left(R_{E}^{p}\right)^{2}}{\left(R_{E}^{p}\right)^{2}+\left(X_{S}^{p}\right)^{2}} \\
Z_{S}^{c p}=\frac{R_{E}^{p} X_{S}^{p}}{\left(R_{E}^{p}\right)^{2}+\left(X_{S}^{p}\right)^{2}}\left[X_{S}^{p}-j R_{E}^{p}\right]
\end{gathered}
$$

Based on the Eq. 42, the real $Z^{p^{\prime}}$ of $Z_{S}^{c p}$ is given by

where the imaginary $Z^{p \prime \prime}$ of $Z_{S}^{c p}$ is given by

$$
Z_{S}^{p^{\prime}}=\frac{R_{E}^{p}\left(X_{S}^{p}\right)^{2}}{\left(X_{S}^{p}\right)^{2}+\left(R_{E}^{p}\right)^{2}}
$$

$$
Z_{S}^{p \prime \prime}=\frac{X_{S}^{p}\left(R_{E}^{p}\right)^{2}}{\left(X_{S}^{p}\right)^{2}+\left(R_{E}^{p}\right)^{2}}
$$

The magnitude or modulus of $Z_{S}^{p}\left(\bmod \left|Z_{S}^{p}\right|\right)$ can be calculated from Eqs. 47 and 48 as follows

$$
\begin{gathered}
\left|Z_{S}^{c p}\right|=\sqrt{\left[\left(\frac{R_{E}^{p}\left(X_{S}^{p}\right)^{2}}{\left(X_{S}^{p}\right)^{2}+\left(R_{E}^{p}\right)^{2}}\right)^{2}+\left(\frac{X_{S}^{p}\left(R_{E}^{p}\right)^{2}}{\left(X_{S}^{p}\right)^{2}+\left(R_{E}^{p}\right)^{2}}\right)^{2}\right]} \\
\left|Z_{S}^{p}\right|=\sqrt{\frac{\left(X_{S}^{p}\right)^{2}\left(R_{E}^{p}\right)^{2}}{\left(X_{S}^{p}\right)^{2}+\left(R_{E}^{p}\right)^{2}}}
\end{gathered}
$$

Now, by using Eq. 38

$$
\left|Z_{S}^{p}\right|=\sqrt{\frac{\left(X_{S}^{p}\right)^{2}\left(R_{E}^{p}\right)^{2}}{\left(V_{S}^{p} / I_{T}^{p} \cos \delta\right)^{2}+\left(R_{E}^{p}\right)^{2}}}
$$

Moreover, the total impedance $\left(Z_{T}^{c p}\right)$ for the circuit show in Figure 3 can be calculated from the following

$$
Z_{T}^{c p}=\sqrt{\left(R_{S}\right)^{2}+2 R_{S}\left|Z_{S}^{p}\right| \cos (90-\delta)+\left(Z_{S}^{p}\right)^{2}}
$$

But

Therefore, Eq. 52 becomes

$$
\cos (90-\delta)=\sin (\delta)
$$

$$
Z_{T}^{c p}=\sqrt{\left(R_{S}\right)^{2}+2 R_{S}\left|Z_{S}^{c p}\right| \sin (\delta)+\left(Z_{S}^{c p}\right)^{2}}
$$

Now, from Eqs. 15 and 54, it can be found the $I_{T}^{p}$

$I_{T}^{p}=\frac{\sqrt{\left(V_{R S}^{P}\right)^{2}+2 V_{R S}^{P} V_{S}^{p} \sin \delta+\left(V_{S}^{p}\right)^{2}}}{\sqrt{\left(R_{S}\right)^{2}+2 R_{S}\left|Z_{S}^{c p}\right| \sin (\delta)+\left(Z_{S}^{c p}\right)^{2}}}$

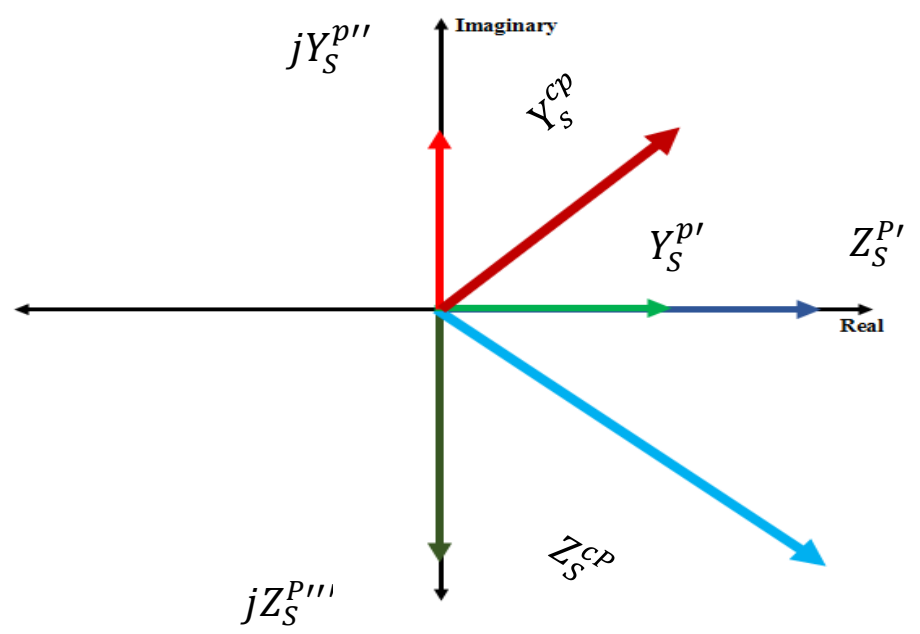

Figure 5: Representation of the $Z_{S}^{c p}$ and the $Y_{s}^{c p}$. 


\section{The admittance function}

In the complex domain, the current $(i=v / Z)$, otherwise, $i=Y v$, with $v$ is the voltage, where all of them are taken as complex quantities. Experimentally, $Z$ and $Y$ can be expressed in terms of resistance and capacitance components. Therefore, the admittance of the examined sample can be given by

Using Eq. 46,

$$
\mathrm{Y}_{S}^{c p} \equiv\left(Z_{S}^{c p}\right)^{-1}
$$

$$
\mathrm{Y}_{S}^{c p}=\left(\frac{\left(R_{E}^{p}\right)^{2}+\left(X_{S}^{p}\right)^{2}}{R_{E}^{p} X_{S}^{p}}\right) \frac{1}{\left(X_{S}^{p}-j R_{E}^{p}\right)}
$$

To simplify the Eq. 57, multiply by conjugate of $\left(X_{S}^{p}-j R_{E}^{p}\right)$

$$
\begin{gathered}
\mathrm{Y}_{S}^{c p}=\left(\frac{\left(R_{E}^{p}\right)^{2}+\left(X_{S}^{p}\right)^{2}}{R_{E}^{p} X_{S}^{p}}\right) \frac{\left(X_{S}^{p}+j R_{E}^{p}\right)}{\left(X_{S}^{p}-j R_{E}^{p}\right)\left(X_{S}^{p}+j R_{E}^{p}\right)} \\
=\frac{1}{R_{E}^{p}}+\frac{j}{X_{S}^{p}}=\frac{1}{R_{E}^{p} X_{S}^{p}}\left(X_{S}^{p}+j R_{E}^{p}\right)
\end{gathered}
$$

However $Y_{S}^{c P}$ is a complex, so it can be expressed by

$$
\mathrm{Y}_{S}^{c p}=\mathrm{Y}_{S}^{p^{\prime}}+j \mathrm{Y}_{S}^{p^{\prime \prime}}
$$

$\mathrm{Y}_{S}^{c s}$ is plotted in Figure 5. As in Eq. 59 the real and imaginary parts of $\mathrm{Y}_{S}^{c p}$ are $1 / R_{E}^{p}$ and $1 / X_{S}^{p}$, respectively. In line with, the $Z_{S}^{p}$ of the parallel $R C$ circuit model is subjected as in Eq. 46, while the $Y_{S}^{c}$ for the parallel $R C$ circuit model, is

$$
\mathrm{Y}_{s}^{c p}=G^{p}+j B^{p}
$$

where the conductance $G^{p}=1 / R_{E}^{p}$ and the susceptance $B^{s}=1 / X_{S}^{p}$. The synonymous unit of Admittance is mho ( $\left.\mho\right)$, and the symbol (an upside-down uppercase omega), are also in common use.

\section{The modulus function}

Other important quantity is the modulus $(M)$, which is, usually, defined by

Using Eq. 58 into Eq. 61, it can be found

$$
\mathrm{M}_{S}^{c p} \equiv \mu\left(\mathrm{Y}_{S}^{c p}\right)^{-1}=M_{S}^{p^{\prime}}+j M_{S}^{p \prime \prime}
$$

$$
\begin{gathered}
\mathrm{M}_{S}^{c p}=\frac{\mu R_{E}^{p} X_{S}^{p}}{\left(X_{S}^{p}+j R_{E}^{p}\right)} \\
\mathrm{M}_{S}^{c p}=\mu R_{E}^{p} X_{S}^{p} \frac{\left(X_{S}^{p}-j R_{E}^{p}\right)}{\left(X_{S}^{p}+j R_{E}^{p}\right)\left(X_{S}^{p}-j R_{E}^{p}\right)} \\
\mathrm{M}_{S}^{c p}=\frac{\mu R_{E}^{p} X_{S}^{p}}{\left(R_{E}^{p}\right)^{2}+\left(X_{S}^{p}\right)^{2}}\left(X_{S}^{p}-j R_{E}^{p}\right)=\mu Z_{S}^{c p}
\end{gathered}
$$

where $\mu \equiv j \omega C^{o}=-1 / j X_{C}^{o}$ with $C^{o}=\varepsilon^{o} A / T$ is the capacitance of the empty measuring cell and $\varepsilon^{o}$ is the dielectric permittivity of free space, $8.854 \times 10^{-12} \mathrm{~F} / \mathrm{m}$. $X_{C}^{o}$ is the capacitive reactance for the empty measuring cell. This implies to the following

$$
\begin{aligned}
\mathrm{M}_{S}^{c p} & =\frac{j \omega C^{o} R_{E}^{p} X_{S}^{p}}{\left(R_{E}^{p}\right)^{2}+\left(X_{S}^{p}\right)^{2}}\left(X_{S}^{p}-j R_{E}^{p}\right) \\
\mathrm{M}_{S}^{c p} & =\frac{\omega C^{o} R_{E}^{p} X_{S}^{p}}{\left(R_{E}^{p}\right)^{2}+\left(X_{S}^{p}\right)^{2}}\left(R_{E}^{p}+j X_{S}^{p}\right)
\end{aligned}
$$

But $C^{o}=1 / X_{C}^{o}$, so Eq. 66, becomes

$$
\mathrm{M}_{S}^{c p}=\frac{R_{E}^{p} X_{S}^{p}}{X_{C}^{o}\left[\left(R_{E}^{p}\right)^{2}+\left(X_{S}^{p}\right)^{2}\right]} R_{E}^{p}+j \frac{R_{E}^{p} X_{S}^{p}}{X_{C}^{o}\left[\left(R_{E}^{p}\right)^{2}+\left(X_{S}^{p}\right)^{2}\right]} X_{S}^{p}
$$

where the real part of the $\mathrm{M}_{S}^{c p}$ is

$$
\begin{gathered}
\mathrm{M}_{S}^{p^{\prime}}=\frac{\omega C^{o} X_{S}^{p}\left(R_{E}^{p}\right)^{2}}{\left[\left(R_{E}^{p}\right)^{2}+\left(X_{S}^{p}\right)^{2}\right]} \\
\mathrm{M}_{S}^{p \prime}=\frac{X_{S}^{p}\left(R_{E}^{p}\right)^{2}}{X_{C}^{o}\left[\left(R_{E}^{p}\right)^{2}+\left(X_{S}^{p}\right)^{2}\right]}
\end{gathered}
$$

and the imaginary part of the $\mathrm{M}_{S}^{c p}$ is

$$
\mathrm{M}_{S}^{p \prime \prime}=\frac{\omega C^{o} R_{E}^{p}\left(X_{S}^{p}\right)^{2}}{\left[\left(R_{E}^{p}\right)^{2}+\left(X_{S}^{p}\right)^{2}\right]}=\frac{R_{E}^{p}\left(X_{S}^{p}\right)^{2}}{X_{C}^{o}\left[\left(R_{E}^{p}\right)^{2}+\left(X_{S}^{p}\right)^{2}\right]}
$$




$$
\mathrm{M}_{S}^{p \prime \prime}=\frac{\omega C^{o} R_{E}^{p}\left(X_{S}^{p}\right)^{2}}{\left[\left(R_{E}^{p}\right)^{2}+\left(X_{S}^{p}\right)^{2}\right]}
$$

\section{Dielectric parameters}

The complex relative dielectric constant or dielectric permittivity $\left(\varepsilon_{r}^{c p}\right)$ is connected to the $\left(\mathrm{M}_{S}^{c p}\right)^{-1}$. From Eq. 66 , the $\varepsilon_{r}^{c p}$ can be expressed by the following

Or one can use Eq. 67

$$
\varepsilon_{r}^{c p}=\frac{\left(R_{E}^{p}\right)^{2}+\left(X_{S}^{p}\right)^{2}}{\omega C^{o} R_{E}^{p} X_{S}^{p}\left(R_{E}^{p}+j X_{S}^{p}\right)}
$$

$$
\varepsilon_{r}^{c p}=\frac{X_{C}^{o}\left[\left(R_{E}^{p}\right)^{2}+\left(X_{S}^{p}\right)^{2}\right]}{R_{E}^{p} X_{S}^{p}} \frac{1}{\left(R_{E}^{p}+j X_{S}^{p}\right)}
$$

The Eq. 72 can be treated to become

$$
\varepsilon_{r}^{c p}=\frac{\left(R_{E}^{p}\right)^{2}+\left(X_{S}^{p}\right)^{2}}{\omega C^{o} R_{E}^{p} X_{S}^{p}} \frac{\left(R_{E}^{p}-j X_{S}^{p}\right)}{\left(R_{E}^{p}+j X_{S}^{p}\right)\left(R_{E}^{p}-j X_{S}^{p}\right)}
$$

The Eq. 73 can be treated to become

$$
\varepsilon_{r}^{c p}=\frac{X_{C}^{o}\left[\left(R_{E}^{p}\right)^{2}+\left(X_{S}^{p}\right)^{2}\right]}{R_{E}^{p} X_{S}^{p}} \frac{\left(R_{E}^{p}-j X_{S}^{p}\right)}{\left(R_{E}^{p}+j X_{S}^{p}\right)\left(R_{E}^{p}-j X_{S}^{p}\right)}
$$

Finally, Eq. 75 becomes

$$
\varepsilon_{r}^{c p}=\frac{1}{\omega C^{o} R_{E}^{p} X_{S}^{p}}\left(R_{E}^{p}-j X_{S}^{p}\right)
$$

Finally, Eq. 76 becomes

$$
\varepsilon_{r}^{c p}=\frac{X_{C}^{o}}{R_{E}^{p} X_{S}^{p}}\left(R_{E}^{p}-j X_{S}^{p}\right)
$$

The complex relative dielectric constant or dielectric permittivity $\left(\varepsilon_{r}^{c p}\right)$ can be expressed by the following

$$
\varepsilon_{r}^{c p}=\varepsilon_{r}^{p \prime}-j \varepsilon_{r}^{p \prime \prime}
$$

From Eqs. 77 and 78

$$
\varepsilon_{r}^{p^{\prime}}=\frac{1}{\omega C^{o} X_{S}^{p}}=\frac{X_{C}^{o}}{X_{S}^{p}}=\frac{C_{S}^{p}}{C^{o}}
$$

Again, from Eqs. 77 and 78

$$
\varepsilon_{r}^{\prime \prime}=\frac{1}{\omega C^{o} R_{E}^{p}}=\frac{X_{C}^{o}}{R_{E}^{p}}
$$

The dielectric loss tangent (dissipation factor) is defined by the relative "lossiness" of a material is the ratio of the energy lost to the energy stored which is given by

$$
\tan \delta=\frac{\varepsilon_{r}^{S^{\prime \prime}}}{\varepsilon_{r}^{S^{\prime}}}
$$

So by using the Eqs. 78 and 79

Again by using the Eqs. 78 and 79

$$
\tan \delta=\frac{X_{C}^{o}}{R_{E}^{p}} \frac{X_{S}^{p}}{X_{C}^{o}}=\frac{X_{S}^{p}}{R_{E}^{p}}
$$

$$
\tan \delta=\frac{X_{C}^{o}}{R_{E}^{p}} \frac{C^{o}}{C_{S}^{p}}
$$

Experimentally, from the phasor diagram the same result can be found by using the Eqs. 35 and 38,

$$
\tan \delta=\frac{X_{S}^{p}}{R_{E}^{p}}
$$

The quality factor or Q-factor" is used with respect to an electronic microwave material, which is the reciprocal of the loss tangent

$$
Q=\frac{1}{\tan \delta}=\frac{R_{E}^{p}}{X_{S}^{p}}=\frac{R_{E}^{p}}{X_{C}^{o}} \frac{C_{S}^{p}}{C^{o}}
$$

\section{The electrical conductivity}


It is, well, known that, the conductivity is conducted as a function with impedance. By using Eq. 50, the magnitude of the electric conductivity of the sample in the parallel model $\left(\sigma_{S}^{p}\right)$ can be expressed as

$$
\begin{gathered}
\sigma_{S}^{p}=\frac{T}{A Z_{S}^{c p}} \\
\sigma_{S}^{p}=\frac{T}{A} \sqrt{\frac{\left(X_{S}^{p}\right)^{2}+\left(R_{E}^{p}\right)^{2}}{\left(X_{S}^{p}\right)^{2}\left(R_{E}^{p}\right)^{2}}}
\end{gathered}
$$

where $T$ and $A$ are the thickness and the cross section area of the examined sample, respectively.

The complex model for the conductivity can be expressed by

Using the Eqs. 46 and 77

$$
\sigma_{S}^{p}=\frac{\varepsilon^{c p}}{C_{S}^{p} Z_{S}^{p}}=\frac{\varepsilon^{o}}{C_{S}^{s}} \frac{\varepsilon_{r}^{c p}}{Z_{S}^{p}}
$$

$$
\begin{gathered}
\sigma_{S}^{p}=\frac{X_{C}^{o} \varepsilon^{o}}{C_{S}^{p}} \frac{\left(R_{E}^{p}\right)^{2}+\left(X_{S}^{p}\right)^{2}}{\left(R_{E}^{p} X_{S}^{p}\right)^{2}}\left(\frac{\left(R_{E}^{p}-j X_{S}^{p}\right)}{\left(X_{S}^{p}-j R_{E}^{p}\right)}\right) \\
\sigma_{S}^{p}=\frac{X_{C}^{o} \varepsilon^{o}}{C_{S}^{p}} \frac{\left(R_{E}^{p}\right)^{2}+\left(X_{S}^{p}\right)^{2}}{\left(R_{E}^{p} X_{S}^{p}\right)^{2}}\left(\frac{\left(R_{E}^{p}-j X_{S}^{p}\right)\left(X_{S}^{p}+j R_{E}^{p}\right)}{\left(X_{S}^{p}-j R_{E}^{p}\right)\left(X_{S}^{p}+j R_{E}^{p}\right)}\right) \\
\sigma_{S}^{p}=\frac{X_{C}^{o} \varepsilon^{o}}{C_{S}^{p}} \frac{1}{\left(R_{E}^{p} X_{S}^{p}\right)^{2}}\left(\left(R_{E}^{p}-j X_{S}^{p}\right)\left(X_{S}^{p}+j R_{E}^{p}\right)\right) \\
\sigma_{S}^{p}=\frac{X_{C}^{o} \varepsilon^{o}}{C_{S}^{p}} \frac{1}{\left(R_{E}^{p} X_{S}^{p}\right)^{2}}\left(2 R_{E}^{p} X_{S}^{p}+j\left[\left(R_{E}^{p}\right)^{2}-j\left(X_{S}^{p}\right)^{2}\right]\right)
\end{gathered}
$$

Therefore, $\sigma_{S}^{p}$ can be expressed by

$$
\sigma_{S}^{p}=\frac{2 X_{C}^{o} \varepsilon^{o}}{C_{S}^{p}} \frac{1}{R_{E}^{p} X_{S}^{p}}+j \frac{X_{C}^{o} \varepsilon^{o}}{C_{S}^{p}} \frac{\left[\left(R_{E}^{p}\right)^{2}-j\left(X_{S}^{p}\right)^{2}\right]}{\left(R_{E}^{p} X_{S}^{p}\right)^{2}}
$$

or

$$
\sigma_{S}^{p}=\frac{2 \varepsilon^{o}}{\omega C^{o} C_{S}^{p}} \frac{1}{R_{E}^{p} X_{S}^{p}}+j \frac{\varepsilon^{o}}{\omega C^{o} C_{S}^{p}} \frac{\left[\left(R_{E}^{p}\right)^{2}-j\left(X_{S}^{p}\right)^{2}\right]}{\left(R_{E}^{p} X_{S}^{p}\right)^{2}}
$$

The conductivity and dipolar relaxation processes may be existence in the same material, and the total conductivity is given by complex electrical conductivity

The real part conductivity is

$$
\sigma_{S}^{p}(\omega)=\sigma_{S}^{p \prime}(\omega)+j \sigma_{S}^{p \prime \prime}(\omega)
$$

$$
\begin{gathered}
\sigma_{S}^{p^{\prime}}(\omega)=\frac{2 X_{C}^{o} \varepsilon^{o}}{C_{S}^{p}} \frac{1}{R_{E}^{p} X_{S}^{p}} \\
=\frac{2 \varepsilon^{o}}{\omega C^{o} C_{S}^{p}} \frac{1}{R_{E}^{p} X_{S}^{p}}
\end{gathered}
$$

The imaginary part of the conductivity is

$$
\begin{gathered}
\sigma_{S}^{p \prime \prime}(\omega)=\frac{X_{C}^{o} \varepsilon^{o}}{C_{S}^{p}} \frac{\left[\left(R_{E}^{p}\right)^{2}-j\left(X_{S}^{p}\right)^{2}\right]}{\left(R_{E}^{p} X_{S}^{p}\right)^{2}} \\
=\frac{\varepsilon^{o}}{\omega C^{o} C_{S}^{p}} \frac{\left[\left(R_{E}^{p}\right)^{2}-j\left(X_{S}^{p}\right)^{2}\right]}{\left(R_{E}^{p} X_{S}^{p}\right)^{2}}
\end{gathered}
$$

\section{Useful relations Conductivity and Immittances Functions}

It was defined that the imaginary part of the conductivity is

$$
\sigma_{S}^{p^{\prime \prime}}(\omega)=\varepsilon_{r}^{p \prime \prime} \varepsilon^{o} \omega
$$

Using Eq. 80 into Eq. 100,

$$
\sigma^{\mathrm{p} \prime \prime}(\omega)=\frac{X_{C}^{o} \varepsilon^{o} \omega}{R_{E}^{p}}=\frac{\varepsilon^{o}}{C^{o} R_{E}^{p}}
$$

Since $C^{o}=A \varepsilon^{o} / T$, so Eq. 101 becomes

$$
\sigma^{\mathrm{p} \prime \prime}(\omega)=\frac{T}{A} \frac{1}{R_{E}^{p}}=\left|\sigma_{S}^{o}\right|\left|Z_{S}^{o}\right| \frac{1}{R_{E}^{p}}
$$

where $\left|\sigma_{S}^{o}\right|=\varepsilon^{o} / C^{o}\left|Z_{S}^{c S}\right|$, is the magnitude for conductivity of the empty of sample. 


\section{CONClusions}

The Delay-Time measurement method can be used to calculate the phase shift $(\varphi)$ between the two different waveforms using an oscilloscope. The sample of the material can be considered as a capacitor in an AC circuit. Different physics quantities were derived and can be calculated by using the Delay-Time measurement method such as immittances, conductivity and dielectric parameters. The presented method, can be, easy, used from the scientists and engineers.

\section{Data Availability}

The data used to support the findings of this study are available from the corresponding author upon request.

\section{Conflicts of Interest}

The author declare that there is no conflict of interest regarding the publication of this paper.

\section{Funding Statement}

The author declare that this research did not receive specific funding, but was performed as part of the employment of the author, Islamic University of Gaza, Palestine. .

\section{ACKNOWLEDGMENTS}

Author would like to acknowledge my colleagues Associate Prof. Hussien Dawoud for his suggestions.

\section{REFERENCES}

[1] Barsoukov, E.; Macdonald, J. R.,L; Impedance Spectroscopy Theory, Experiment, and Applications; Second Edition, John Wiley \& Sons, Inc., Hoboken; New Jersey; 2005.

[2] Sinclair, D. C.; Bol. Soc, Esp. Cerám. Vidrio, 1995, 34(2), 55-65.

[3] Barik, S. K.; Choudhary, R. N. P.; Singh,A. K.; Adv. Mat. Lett., 2011, 2(6), 419-424,.

[4] Pavlović, M. M.; Pavlović, M. G.; Panić, V.; Talijan. N.; L. Vasiljević, M. V. Tomić, Int. J. Electrochem. Sci., 7, 2012, 8894 - 8904.

[5] Parasitic, http://bwrcs.eecs.berkeley.edu/Classes/icdesign/ee141_f01/Notes/chapter4.pdf. Accessed on 22/01/2017,

[6] Yangang Wang and M. Zwolinski, DOI: 10.1109/ISCAS.2009.5118433 · Source: IEEE ExploreL.

[7] J. R. Burns, RCA Rev., vol. 25, pp. 627-661, Dec. 1964.

[8] A. A. Hamoui, and N. C. Rumin, IEEE Trans. Circuits and systems-II, 47(10). 999-1007, 2000.

[9] N. Hedenstierna, and K. O. Jeppson, 6(2), 270-281, 1987.

[10] B. Cline, K. Chopra, D. Blaauw, A. Torres, and S. Sundareswaran, Design, automation and test in Europe, 592-597, 2008.

[11] L. Z. Zhang, W. Chen, Y. Hu, and C. C. Chen, IEEE Trans. Computer-Aided Design, 25(6), 1183-1191, 2006.

[12] T. Sakurai and A. R. Newton, IEEE J. Solid-state circuits, vol. 25(2), 584-594, 1990.

[13] K. Mazloomi, N. Sulaiman, H. Moayedi, Int. J. Electrochem. Sci., 7 (2012) 3466 - 3481.

[14] Phase angle measurements, http://eeshop.unl.edu/pdf/OscilloscopeTutorial--PhaseMeasurement.pdf. accessed on 22/01/2017,

[15] Samy K. K. Shaat and Hussein A. Dawoud, Advanced Materials Proceedings 2017, 2(10), 615-620. 